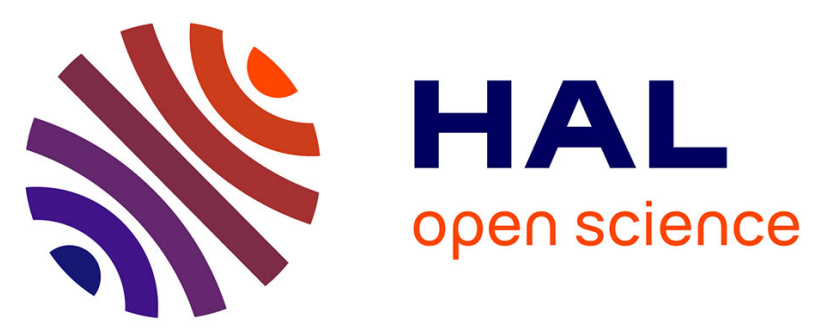

\title{
Classification of Very High Resolution SAR Images of Urban Areas Using Copulas and Texture in a Hierarchical Markov Random Field Model
}

Aurélie Voisin, Vladimir A. Krylov, Gabriele Moser, Sebastiano B. Serpico, Josiane Zerubia

\section{To cite this version:}

Aurélie Voisin, Vladimir A. Krylov, Gabriele Moser, Sebastiano B. Serpico, Josiane Zerubia. Classification of Very High Resolution SAR Images of Urban Areas Using Copulas and Texture in a Hierarchical Markov Random Field Model. IEEE Geoscience and Remote Sensing Letters, 2013, 10 (1), pp.96-100. 10.1109/LGRS.2012.2193869 . hal-00723280

\section{HAL Id: hal-00723280 https://hal.inria.fr/hal-00723280}

Submitted on 8 Aug 2012

HAL is a multi-disciplinary open access archive for the deposit and dissemination of scientific research documents, whether they are published or not. The documents may come from teaching and research institutions in France or abroad, or from public or private research centers.
L'archive ouverte pluridisciplinaire HAL, est destinée au dépôt et à la diffusion de documents scientifiques de niveau recherche, publiés ou non, émanant des établissements d'enseignement et de recherche français ou étrangers, des laboratoires publics ou privés. 


\title{
Classification of Very High Resolution SAR Images of Urban Areas Using Copulas and Texture in a Hierarchical Markov Random Field Model
}

\author{
Aurélie Voisin, Vladimir A. Krylov, Gabriele Moser, Member, IEEE, Sebastiano B. Serpico, Fellow, IEEE, \\ and Josiane Zerubia, Fellow, IEEE
}

\begin{abstract}
This letter addresses the problem of classifying synthetic aperture radar (SAR) images of urban areas by using a supervised Bayesian classification method via a contextual hierarchical approach. We develop a bivariate copula-based statistical model that combines amplitude SAR data and textural information, which is then plugged into a hierarchical Markov random field model. The contribution of this letter is thus the development of a novel hierarchical classification approach that uses a quad-tree model based on wavelet decomposition and an innovative statistical model. The performance of the developed approach is illustrated on a high-resolution satellite SAR image of urban areas.
\end{abstract}

Index Terms-Hierarchical Markov random fields (MRFs), supervised classification, synthetic aperture radar (SAR), textural features, urban areas, wavelets.

\section{INTRODUCTION}

$\mathbf{S}$ YNTHETIC aperture radar (SAR) is an active image acquisition system which allows day-and-night and all-weather acquisitions [1]. Such properties are crucial, for example, for the risk management application, allowing land use and land cover mapping, as well as the detection of areas damaged by natural disasters such as earthquakes or floodings. In this letter, we address the problem of classifying SAR images of urban areas, which represents a particularly interesting typology in important applications such as civil protection for disaster monitoring and damage assessment.

Several difficulties need to be considered to address the SAR classification problem. The first one is related to the inherent multiplicative noise known as speckle, which originates from the interference of the coherent wavefronts. This type of noise is specific to active imaging systems and degrades appreciably the registered imagery [1]. As a consequence, standard classification methods that have been validated for optical data

Manuscript received February 27, 2012; revised March 28, 2012; accepted March 29, 2012. The work of A. Voisin was supported in part by the Direction Générale de l'Armement (France) and in part by the Institut National de Recherche en Informatique et Automatique (France).

A. Voisin, V. A. Krylov, and J. Zerubia are with the Ayin Team, Institut National de Recherche en Informatique et Automatique, 06902 Sophia Antipolis, France (e-mail: Aurelie.Voisin@inria.fr; Vladimir.Krylov@inria.fr; Josiane.Zerubia@inria.fr).

G. Moser and S. B. Serpico are with the Dipartimento di Ingegneria Biofisica ed Elettronica, University of Genoa, 16145 Genoa, Italy (e-mail: gabriele.moser@unige.it; sebastiano.serpico@unige.it).

Color versions of one or more of the figures in this paper are available online at http://ieeexplore.iee.org.

Digital Object Identifier 10.1109/LGRS.2012.2193869 do not report satisfying results when applied directly to SAR images. Another difficulty is the heterogeneity of urban areas in very high resolution (VHR) images that leads to heterogeneous statistical modeling, reflecting different ground materials such as asphalt, concrete, and metal.

A variety of supervised methods have been considered for SAR image classification in the literature, including various techniques such as active contours [2], bags of words [3], and rule-based methods [4].

In this letter, we propose a statistical Bayesian supervised classification approach that consists of two steps. The first step deals with the SAR amplitude statistical modeling for each target class (e.g., vegetation and urban) by using finite mixtures of automatically chosen SAR-specific probability density functions (pdfs) [5], which are intended to take into account the aforementioned VHR SAR statistics heterogeneity. We further consider an additional source of information obtained by extracting a textural feature map from the original SAR image in order to optimize the detection of urban areas. The marginal pdfs of the original SAR image and the textural feature are combined via copulas [6], leading to a joint pdf for each class. In the second step, the classification map is generated, using the joint copula-based statistics. To improve the robustness with respect to speckle noise, we consider a contextual model based on a hierarchical Markov random field (MRF) [7].

The novel contribution of this letter is twofold. First, we propose a joint amplitude-texture pdf modeling that aims to help discriminate the urban areas. Second, we integrate this joint pdf model into a hierarchical MRF in which we introduce a prior estimation update that experimentally leads to improved results, which are less affected by speckle noise when compared to a predefined prior model [8].

This letter is organized as follows. In Section II, we introduce the statistical bivariate copula-based model that combines the marginal pdfs of SAR amplitude data and its textural feature. In Section III, we develop a marginal posterior mode (MPM) model based on a quad tree that integrates a prior update. In Section IV, we present the classification results obtained in a high-resolution COSMO-SkyMed image. Finally, in Section V, we draw conclusions.

\section{Joint Amplitude-TeXture PDF Model}

To construct an urban-area-specific model, we propose to extract additional textural information from the original SAR 
image. To this end, the class-conditional marginal statistics of the SAR image and its textural feature are independently modeled and then gathered into a joint amplitude-texture pdf by using the statistical instrument of copulas.

\section{A. Textural Features}

Well-chosen textural features turn out to be discriminative with respect to urban areas. Taking into account this information is helpful when classifying such areas. Motivated by the experimental textural feature extraction study performed in [9], we consider a feature given by gray-level co-occurrence matrices (GLCMs) [10]. It describes the joint statistics of the gray levels of different pixels as a function of their reciprocal locations. Typically, the element $(i, j)$ of the matrix is the probability that a pixel with value $i$ is adjacent, in a given direction, to a pixel with value $j$. In our case, we consider horizontal adjacency, with an offset equal to one, i.e., the cooccurrence matrix is filled by considering a reference pixel and the pixel located to its right. Among various textures that can be extracted from the GLCM, we use the variance, which discriminates well the urban areas (as confirmed by our experiments).

Such approach to texture extraction is applied on a movingwindow basis, meaning that each pixel of the image successively becomes the central pixel of the window, and its value is replaced by the variance value estimated within the window. According to preliminary experiments, the choice of a $5 \times 5$ local window provides more accurate results on the considered data sets than any other window of size in the range [3; 17]. Higher values of window size have not been considered so as to avoid an smearing effect on object edges.

\section{B. Combined Amplitude-Texture Model}

We now present the marginal statistics estimation and the construction of a copula-based joint amplitude-texture pdf $p\left(y \mid \omega_{m}\right)$. Here, $y=\left(y_{1}, y_{2}\right)$ represents the original image $y_{1}$ and its corresponding textural feature $y_{2} ; \omega_{m}$ is the $m$ th class considered for the classification, with $m \in[1 ; M]$.

1) Marginal PDF Estimation: Given a training set, for each input image $j$ (SAR and textural feature) and for each class $m$, the pdf $p_{m}\left(y_{j} \mid \omega_{m}\right)$ is modeled by finite mixtures of independent gray-level distributions

$$
p_{m}\left(y_{j} \mid \omega_{m}\right)=\sum_{i=1}^{K} P_{m i} p_{m i} t\left(y_{j} \mid \theta_{m i}\right)
$$

where $P_{m i}$ represents the mixing proportions such that, for a given $m, \sum_{i=1}^{K} P_{m i}=1$ with $0 \leq P_{m i} \leq 1$, and $\theta_{m i}$ is the set of parameters of the $i$ th pdf mixture component of the $m$ th class. The use of finite mixtures instead of single pdfs offers the possibility to consider heterogeneous pdfs, usually reflecting the additive contributions of the different materials present in each class (for instance, different kinds of crops for the vegetation class). Such class heterogeneity is relevant since we deal with VHR images [5].

The pdfs $p_{m i}\left(y_{j} \mid \theta_{m i}\right)$ are automatically chosen in a predefined dictionary including the following four distributions: lognormal, Weibull, Nakagami, and generalized gamma. To estimate the best fitting mixture model for each considered class, we combine a density parameter estimation via the method of $\log$ cumulants and a stochastic expectation-maximization algorithm (see [5] and [11]).

The presented estimation approach was initially developed for SAR amplitude data, but the high flexibility of this model and the lack of accurate statistical model for the textural data motivated us to extend its use to the extracted textural features. We empirically observed the very high estimation quality of this approach.

2) Copula-Based Joint PDF Modeling: The use of the copula instrument allows us to model joint pdfs for amplitude and textural SAR channels by fitting several different dependence structures and selecting the best fit. A bivariate copula is a 2-D joint distribution defined on $[0,1]^{2}$ such that marginal distributions are uniform on $[0,1]$. According to Sklar's theorem [6], we can obtain a unique class-conditional joint pdf $p\left(y \mid \omega_{m}\right)$ for any continuous random variables $y_{1}$ and $y_{2}$ in $\mathbb{R}^{+}$as

$p_{m}\left(y_{1} \mid \omega_{m}\right) p_{m}\left(y_{2} \mid \omega_{m}\right) \times \frac{\partial^{2} C_{m}}{\partial y_{1} \partial y_{2}}\left(F_{m}\left(y_{1} \mid \omega_{m}\right), F_{m}\left(y_{2} \mid \omega_{m}\right)\right)$

where $p_{m}\left(y_{1} \mid \omega_{m}\right)$ and $p_{m}\left(y_{2} \mid \omega_{m}\right)$ are the marginal pdfs estimated in Section II-B1 and $\left\{F_{m}\right\}$ represents their corresponding cumulative distribution functions. For each class $m$, the computation of the joint pdf $p\left(y \mid \omega_{m}\right)$ boils down to the determination of the copula family $C_{m}$ according to (2).

To find the best fitting copula $C_{m}$, we consider a dictionary of five copulas: Clayton, Ali-Mikhail-Haq, Frank, Marchal-Olkin, and Farlie-Gumbel-Morgenstern [6]. This choice of copulas is somewhat arbitrary but is capable of modeling a wide variety of dependence structures [6], [12]. This set of copulas can be adjusted to fit specific classes for a given application. The analytical expressions of these copulas involve only one parameter $\alpha_{m}$. To estimate this parameter, we use the relationship between copulas and Kendall's $\tau$, a correlation coefficient [6] that can be empirically estimated over a training set. Then, for each class $m$, we choose the best fitting copula of the dictionary according to the highest $p$ value reported by the Pearson chi-square goodness-of-fit test [11].

\section{DeVEloped HierarchicAl CLASSIFICATION APPROACH}

We aim at estimating a set of hidden labels $X$ given a set of observations $Y . X$ and $Y$ are considered to be random processes, and $X$ is Markovian with respect to scale. We propose to employ an explicit hierarchical graph-based model [8] to address our classification problem. In the following, we shall focus on specific graphs, which have a quad-tree structure (see Fig. 1). The set of sites $s$ is, therefore, hierarchically partitioned on $S=S^{0} \cup S^{1} \cup \cdots \cup S^{R}$, where $R$ corresponds to the coarsest resolution (the root) and 0 is the reference level (the finest resolution).

The consideration of a quad tree allows benefiting from its good properties (e.g., causality) and applying noniterative algorithms, resulting in a computational time decrease. Among the different algorithms employed in the literature, a first option 


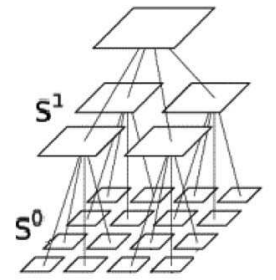

(a)

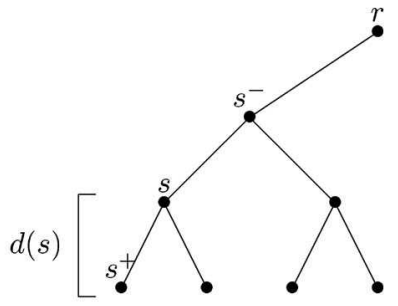

(b)
Fig. 1. (a) Hierarchical model structure: Quad tree. (b) Quad-tree notations.

is to estimate exactly the maximum a posteriori probability, but such a criterion is known to generate underflow by the consideration of very small probabilities. For this reason, we take into account an exact estimator of the MPM [8]. The cost function associated to this estimator offers the possibility to penalize the errors according to their number and the scale at which they occur: For example, an error at the coarsest scale is more strongly penalized than an error at the finest scale.

\section{A. Posterior Probabilities and Their Estimation Using MPM}

The aim here is to maximize the posterior marginal at each site $s$. A classical MPM algorithm [8] is generally run in two passes, referred to as bottom-up ("forward") and top-down ("backward") passes. The former estimates the partial posterior marginals $p\left(x_{s} \mid y_{d(s)}\right)$, and the latter estimates successively the labels at each tree level $n$ by maximizing the posterior marginals $p\left(x_{s} \mid y\right)$. Laferte et al. [8] showed that

$p\left(x_{s} \mid y_{d(s)}\right) \propto p\left(y_{s} \mid x_{s}\right) p\left(x_{s}\right) \prod_{t \in s^{+}} \sum_{x_{t}}\left[\frac{p\left(x_{t} \mid y_{d(t)}\right)}{p\left(x_{t}\right)} p\left(x_{t} \mid x_{s}\right)\right]$.

Thus, the bottom-up pass is a recursion that estimates $p\left(x_{s} \mid y_{d(s)}\right)$ starting from the leaves and proceeding until the root is reached. The needed prior data are the likelihood (see Section II), the prior probabilities (see Section III-C), and the transition probabilities (see Section III-B).

In the proposed algorithm, the top-down pass is truncated, and we only maximize the posterior marginal at the root (see Figs. 2 and 3). The obtained classification map is thus used to update the prior probabilities at this level (Section III-C). The prior distribution at levels $n \in[0 ; R-1]$ is given by

$$
p\left(x_{s}^{n}\right)=\sum_{x_{s^{-}}^{n} \in \Omega=\left\{\omega_{i}, i=1, \ldots, M\right\}} p\left(x_{s}^{n} \mid x_{s^{-}}^{n}\right) p\left(x_{s^{-}}^{n}\right) .
$$

Then, a novel MPM algorithm is run on a smaller quad tree until scale 0 is reached. Such procedure aims to improve the robustness with respect to the speckle noise [13].

The $p\left(x_{s} \mid y\right)$ maximization is done by employing a modified Metropolis dynamics algorithm, which has good properties for both its relative low computation time and the good precision of its results [14].

\section{B. Transition Probabilities}

The transition probabilities between the scales $p\left(x_{s} \mid x_{s^{-}}\right)$determine the hierarchical MRF since they represent the causality of the statistical interactions between the different levels of the
WAVELET TRANSFORM

QUAD-TREE
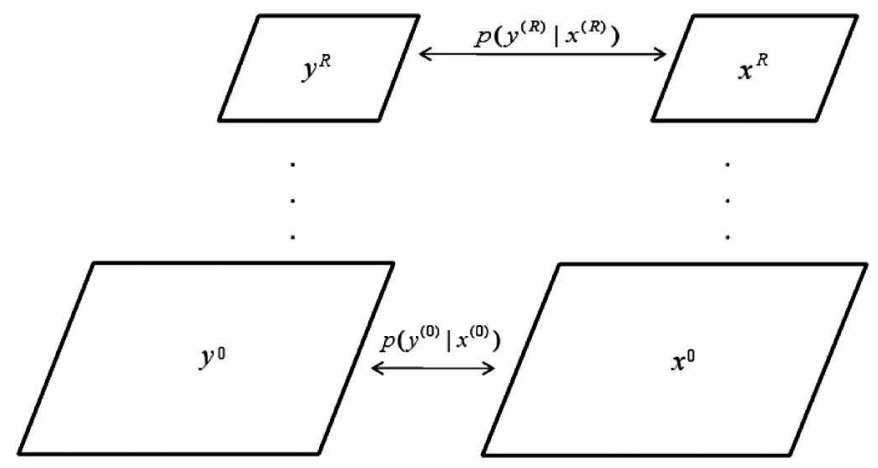

Fig. 2. Generic hierarchical graph-based model of the quad tree.

tree. We use the transition probability in the form introduced by Bouman et al., used in [8]: For all sites $s \in S$ and all scales $n \in[0 ; R-1]$

$$
p\left(x_{s}=\omega_{m} \mid x_{s^{-}}=\omega_{k}\right)= \begin{cases}\theta_{n}, & \text { if } \omega_{m}=\omega_{k} \\ \frac{1-\theta_{n}}{M-1}, & \text { otherwise }\end{cases}
$$

where $\omega_{m}$ and $\omega_{k}$ represent the classes $m$ and $k$, respectively, with $m, k \in[1 ; M]$, and $M$ represents the number of considered classes for the final classification. $\theta_{n}$ is the prior parameter with $\theta_{n}>1 / M$, here chosen as independent of the tree level $n$. This model favors an identical parent-child labeling.

\section{Prior Probabilities}

To estimate the prior probabilities given the obtained classification map, we resort to a single-scale Markovian model which takes into account the spatial contextual information, which therefore leads to a better prior estimation. By employing the Hammersley-Clifford theorem, we can define a local characteristic for each site

$$
p\left(x_{s}\right)=\frac{1}{Z} \exp \left(-\beta \sum_{s:\{s, t\} \in C} \delta_{x_{s}=x_{t}}\right)
$$

where $\delta_{x_{s}=x_{t}}=1$ if $x_{s}=x_{t}$ and $\delta_{x_{s}=x_{t}}=0$ if otherwise and where $Z$ is the normalization constant, $s$ and $t$ denote the sites in the same clique, and $x_{s}$ and $x_{t}$ are their labels. Instead of using a second-order neighborhood set based on the eight pixels surrounding a given pixel, we consider different kinds of set geometries (adaptive neighborhood), and we select the one which leads to the smallest energy at a given site [13]. The adaptivity of the neighborhood aims to take into account the geometrical properties of the different areas in our original image.

\section{EXPERIMENTAL RESUlTS}

We present the results obtained on a $920 \times 820$ pixels singlepolarization COSMO-SkyMed image of the quay of Port-auPrince (Haiti) (copyright Agenzia Spaziale Italiana, 2009), shown in Fig. 4(a), with the following characteristics: $\mathrm{HH}$ polarization, stripmap acquisition mode (2.5-m pixel spacing), 


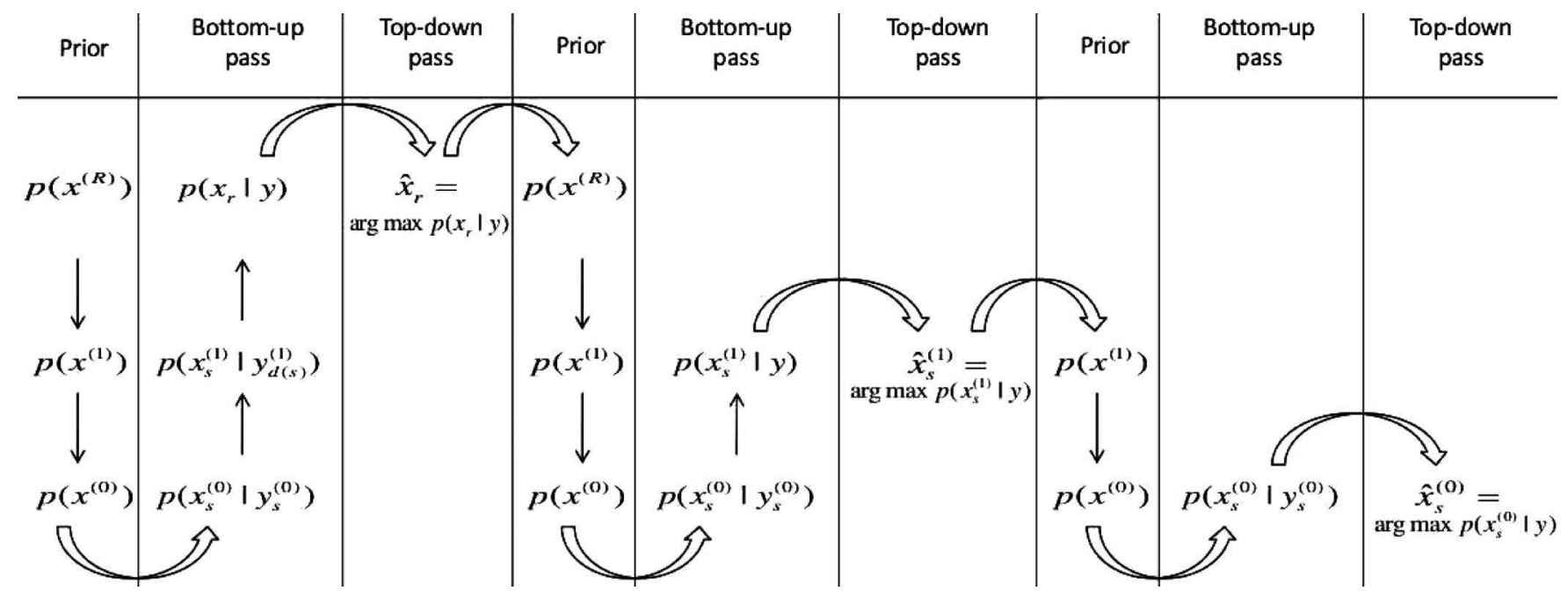

Fig. 3. Proposed MPM estimation on the quad tree shown in Fig. 2. In this representation, $R=2$.

(a)

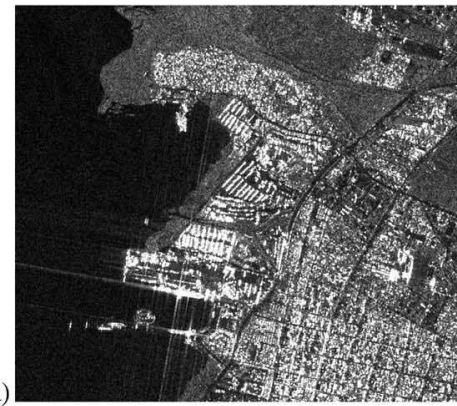

(d)

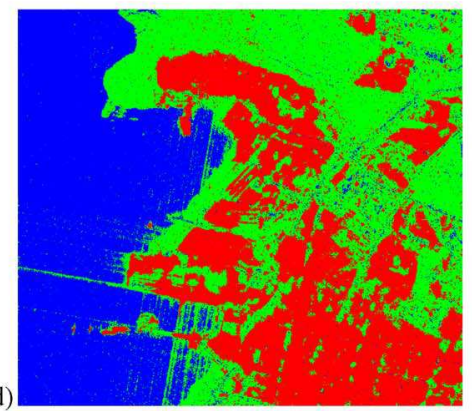

(b)
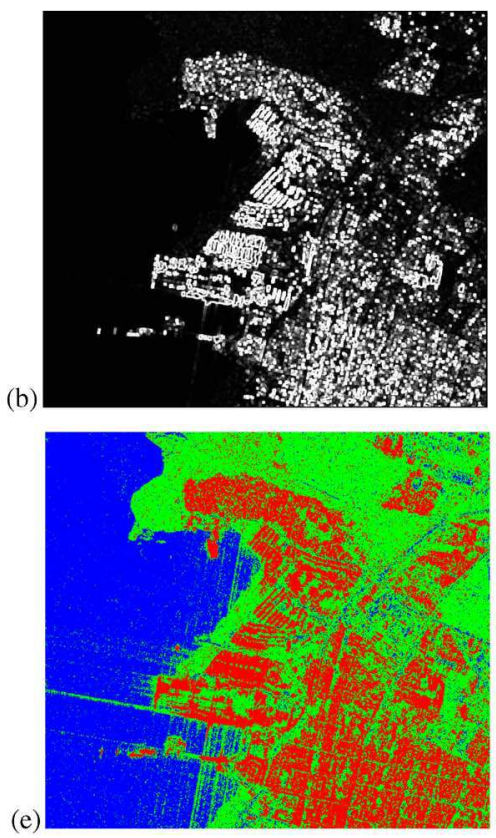
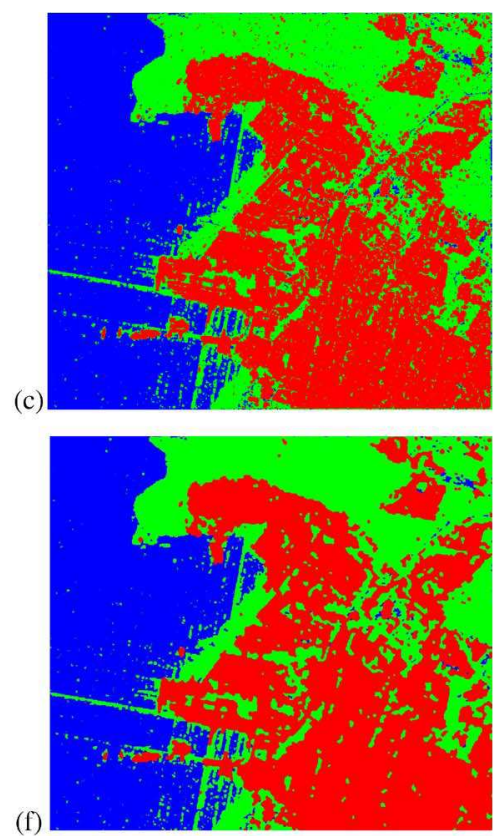

Fig. 4. Port-au-Prince data set. (a) SAR input image and (b) its extracted textural feature. Classification maps (water in blue, urban areas in red, and vegetation in green) obtained applying different methods: (c) Amplitude-texture statistics combined with the hierarchical method proposed in this letter, (d) hierarchical method proposed in this letter based solely on amplitude data, (e) Laferte's hierarchical method based on amplitude data, and (f) amplitude-texture statistics combined with a single-scale MRF model.

geocoded, and single-look image. In the considered explicit hierarchical model, we need multiscale input data. When, as in our case, a multiresolution input is not available, we suggest to decompose our image by resorting to a 2-D discrete wavelet transform. The approximation coefficients at each scale are considered as a multiresolution input. At each level, the textural feature map is obtained from the corresponding image in the decomposition stack. Finally, at each level, the wavelet image is combined with the textural image via a copula-based model presented in Section II. Empirically, we selected the Daubechies10 wavelet [15] that reported the highest classification accuracy when compared to the other families [13].

We built nonoverlapping training and test sets and compared the following classification methods:

1) the proposed amplitude-texture MPM-based approach;
2) the proposed MPM-based approach based solely on the amplitude statistical model (no textures);

3) Laferte's hierarchical MRF approach based solely on the amplitude statistical model (no textures);

4) the amplitude-texture statistical model (Section II) combined with a single-scale MRF (parameter set to $\beta=1.2$ ).

Laferte's algorithm is applied to a quad tree [8], and the prior probabilities at the root are estimated by using a $K$-nearest neighbor [16] preliminary classification, with $K$ obtained by cross-validation. The considered classes are water, urban areas, and vegetation. Empirically, we have concluded that good results are obtained with the decomposition on $R=2$ levels, $\beta=4.8(5)$, and $\theta_{n}=0.8(5)$. 
This article has been accepted for inclusion in a future issue of this journal. Content is final as presented, with the exception of pagination.

TABLE I

ACCURACY FOR EACH OF THE ThreE Classes and Overall Results FOR THE TEST AREAS OF THE PORT-AU-PRINCE QUAY

\begin{tabular}{|l||l|l|l|l|}
\hline \multicolumn{1}{|c||}{} & \multicolumn{4}{c|}{ Port-au-Prince quay } \\
\hline \hline & water & urban & vegetation & overall \\
\hline Proposed method & $97.65 \%$ & $97.99 \%$ & $98.56 \%$ & $\mathbf{9 8 . 0 7 \%}$ \\
\hline Proposed method (no texture) & $97.67 \%$ & $97.27 \%$ & $98.29 \%$ & $\mathbf{9 7 . 7 4 \%}$ \\
\hline Laferte (no texture) & $97.61 \%$ & $88.52 \%$ & $96.74 \%$ & $\mathbf{9 4 . 2 9 \%}$ \\
\hline MRF-based classif. & $97.59 \%$ & $99.03 \%$ & $99.28 \%$ & $\mathbf{9 8 . 6 3 \%}$ \\
\hline
\end{tabular}

The results obtained by our proposed method are satisfying: The classification map [Fig. 4(c)] is quite detailed, and the accuracy table (Table I) indicates that the overall accuracy is $98.07 \%$. As expected, the hierarchical model allows taking into account spatial details and is also relatively robust to speckle noise. Moreover, the urban areas are well discriminated by the use of the textural information in the appropriate copula-based model. Such improvement is shown when comparing the results obtained by the proposed method applied to only amplitude SAR image and applied to the amplitude-texture combination [see Fig. 4(c) and (d)].

To isolate the improvements brought by our proposed hierarchical model, we compare it with Laferte's method, with both based only on the amplitude statistical model. The former model is less affected by speckle noise (Fig. 4) owing to the prior update, particularly for the vegetation class (in the top center of the maps). Moreover, the urban classification accuracy is higher with the developed method. The Laferte one classifies almost all roads as vegetation [e.g., in the bottom center of Fig. 4(e)].

By looking at Table I, we notice that slightly better results for this data set are obtained with the single-scale MRF-based approach [Fig. 4(f)]. We stress though that this result is obtained at the expense of a quite strong smearing effect on object borders (this does not affect the classification accuracy in Table I because the test set has been extracted from the internal parts of objects). Thus, the hierarchical approach is preferable for the urban area classification since it allows extracting more details than the single-scale model. In general, the main water misclassifications come from the crosslike artifact of the SAR acquisition (the point spread function of the SAR sensor [1]). We observe that none of the algorithms employed in this comparison is robust to such artifacts.

Experiments were conducted on an Intel Xeon quad-core (2.40 GHz and 12-MB cache) 18-GB-RAM 64-bit Linux system. Our proposed algorithm runs in $6 \mathrm{~min}$ and $45 \mathrm{~s}$, which is acceptable when compared to the Laferte method computation time of $5 \mathrm{~min}$ and $30 \mathrm{~s}$, given the visual refinement of the classification map.

\section{CONCLUSION}

The supervised classification method proposed in this letter combines a joint copula-based statistical modeling of amplitude SAR images and extracted textural features, with a hierarchical MRF that integrates a prior probability update, thus improving the robustness of the classifier with respect to speckle noise. The labels are determined by resorting to a noniterative optimization algorithm (MPM estimation). The results of the proposed hierarchical classification algorithm appear to be a good tradeoff between the quite blurred results produced by the single-scale MRF-based method and the rather noisy ones obtained by using the Laferte standard model. Furthermore, the developed approach can be extended to the use of multipolarization, multiresolution, and/or multisensor data, which represents a crucial advantage given the considerable amount of remotely sensed data acquired on a daily basis. This is considered as the main direction of further research.

\section{ACKNOWLEDGMENT}

The authors would like to thank Dr. M. De Martino (University of Genoa, Genoa, Italy) for preparing the ground truth maps and the Agenzia Spaziale Italiana for providing the COSMOSkyMed (CSK) image (COSMO-SkyMed Product—copyright Agenzia Spaziale Italiana-2009. All Rights Reserved) in the framework of the project "Development and validation of multitemporal image analysis methodologies for multirisk monitoring of critical structures and infrastructures (2010-2012)."

\section{REFERENCES}

[1] C. Oliver and S. Quegan, Understanding Synthetic Aperture Radar Images. New Delhi, India: SciTech Publ., 2004.

[2] M. Silveira and S. Heleno, "Separation between water and land in SAR images using region-based level sets," IEEE Geosci. Remote Sens. Lett., vol. 6, no. 3, pp. 471-475, Jul. 2009.

[3] J. Feng, L. Jiao, X. Zhang, and D. Yang, "Bag-of-visual-words based on clonal selection algorithm for SAR image classification," IEEE Geosci. Remote Sens. Lett., vol. 8, no. 4, pp. 691-695, Jul. 2011.

[4] S. Dellepiane, D. D. Giusto, S. B. Serpico, and G. Vernazza, "SAR image recognition by integration of intensity and textural information," Int. J. Remote Sens., vol. 12, no. 9, pp. 1915-1932, 1991.

[5] G. Moser, S. B. Serpico, and J. Zerubia, "Dictionary-based stochastic expectation maximization for SAR amplitude probability density function estimation," IEEE Trans. Geosci. Remote Sens., vol. 44, no. 1, pp. 188200, Jan. 2006.

[6] R. B. Nelsen, An Introduction to Copulas, 2nd ed. New York: SpringerVerlag, 2006.

[7] C. Graffigne, F. Heitz, P. Perez, F. Preteux, M. Sigelle, and J. Zerubia, "Hierarchical Markov random field models applied to image analysis: A review," in Proc. SPIE Conf. Neural, Morphological Stochastic Methods Image Process., 1995, vol. 2568, pp. 2-17.

[8] J.-M. Laferte, P. Perez, and F. Heitz, "Discrete Markov modeling and inference on the quad-tree," IEEE Trans. Image Process., vol. 9, no. 3, pp. 390-404, Mar. 2000.

[9] A. Voisin, G. Moser, V. Krylov, S. B. Serpico, and J. Zerubia, "Classification of very high resolution SAR images of urban areas by dictionarybased mixture models, copulas and Markov random fields using textural features," in Proc. SPIE Symp. Remote Sens., Toulouse, France, Sep. 2010, vol. 7830, p. 783000 .

[10] R. M. Haralick, K. Shanmugam, and I. Dinstein, "Textural features for image classification," IEEE Trans. Syst., Man, Cybern., vol. SMC-3, no. 6, pp. 610-621, Nov. 1973.

[11] V. Krylov, G. Moser, S. B. Serpico, and J. Zerubia, "Supervised high resolution dual polarization SAR image classification by finite mixtures and copulas," IEEE J. Sel. Topics Signal Proc., vol. 5, no. 3, pp. 554-566, Jun. 2011.

[12] D. Huard, G. Evin, and A.-C. Fabre, "Bayesian copula selection," Comput. Stat. Data Anal., vol. 51, no. 2, pp. 809-822, Nov. 2006.

[13] A. Voisin, V. Krylov, G. Moser, S. B. Serpico, and J. Zerubia, "Classification of very high resolution SAR images of urban areas," INRIA, Paris, France, Res. Rep. 7758, Oct. 2011.

[14] M. Berthod, Z. Kato, S. Yu, and J. Zerubia, "Bayesian image classification using Markov random fields," Image Vis. Comput., vol. 14, no. 4, pp. 285 295, May 1996.

[15] I. Daubechies, "Orthonormal bases of compactly supported wavelets," Commun. Pure Appl. Math., vol. 41, no. 7, pp. 909-996, Oct. 1988.

[16] C. M. Bishop, Pattern Recognition and Machine Learning. New-York: Springer-Verlag, 2006. 